\title{
Opinião
}

\section{A ESTRATÉGIA DO PROGRAMA SAÚDE DA FAMILIA}

Lívia Arcanjo Fonteles (1) Francisca Ergovânia Batista de Brito (2) Emmanuel Nunes Sampaio de Lacerda (3) Agrênia Custódio de Lima (4)

\section{Resumo}

A Saúde coletiva tem sido um dos principais objetivos da estratégia da Saúde da Família. Este Programa vem executando suas ações numa perspectiva transdisciplinar, favorecendo um projeto assistencial que busca atender e minimizar as necessidades de saúde da comunidade onde atua. O presente estudo pretende discutir este tipo de estratégia, tornando explícito o papel dos envolvidos, na construção deste modelo assistência comunitária em saúde.

Palavras-chave: Saúde da família, Programa Assistencial, Saúde Comunitária

\section{Introdução}

A importância do trabalho em equipe nos Programas de Saúde da Família, é sempre evidenciado, pois trata-se de um trabalho, onde o principal aspecto integrador são os cuidados de saúde. Atualmente esta estratégia é um dos princípios do Sistema Único de Saúde (SUS). No início do Século houve uma grande preocupação com consolidação de um novo modelo de atenção à saúde no Brasil. Para alguns autores (MATTOS, 2001, 2003; CECÍCLIO, 2001), este modelo deveria ser repensado, de forma a com a concepção de integralidade como um pois a saúde precisava incluir aspectos subjetivos resultantes da interação entre os envolvidos.

Rocha (1997) afirma que após a consolidação da Constituição Brasileira, por volta de 1988 em diante, foi um período de tensão entre o SUS e o modelo neoliberal na área da saúde representado entre outros aspectos pela redução de gastos públicos, incentivos às atividades privadas e de mercado. O projeto reformador da saúde, consolidado na Reforma Sanitária brasileira, deparou-se assim, no início dos 90, com o forte conflito entre os desejos de mudanças oriundos de sua proposta original e a dura realidade política-econômica-social do período, o que resultou em maiores dificuldades para a implementação do sistema.

O Programa Saúde da Família - PSF foi criado em 1994, e já nos seus primeiros anos de existência foi marcado por inquietações e críticas à sua proposta (PAIM, 2001). 
À partir daí, foram se incorporando novas propostas, normatizações e novas práticas, e o SUS passou por uma grande reestruturação, principalmente na Atenção Básica. O trabalho, mais humano, passou a centrar-se na clientela, na integralidade das ações dos atores e incluiu o acolhimento, as visitas domiciliares e as ações baseadas no consenso da equipe multiprofissional (BRASIL, 2001).

A obtenção de impactos positivos sobre os diferentes fatores que interferem no processo saúde-doença, passaram a ser a meta da equipe, sendo considerados transformadores da realidade comunitária.

Assim, a abordagem integral dos indivíduos e famílias ficou facilitada pela soma dos distintos olhares profissionais. Mas, a estruturação do trabalho em equipes multiprofissionias no PSF (Programa de Saúde da Família), por si só, não garante uma ruptura com à dinâmica médico-centrada; para tanto, há necessidade de dispositivos que alterem a dinâmica do trabalho em saúde, nos fazeres do cotidiano de cada profissional (FRANCO e MERHY, 2000). Há que se identificar, nessas equipes, os elementos que configurariam uma nova lógica no agir desses profissionais e na forma como se produz o cuidado em saúde. Nesse sentido, o perfil dos recursos humanos em saúde deve alterar-se. Uma das maiores dificuldades na implementação da ESF diz respeito à carência de profissionais para atender a esta nova realidade (CAMPOS e BELISÁRIO, 2001). Faz-se necessário, portanto, o desenvolvimento de um processo de formação e capacitação permanente de todos os profissionais envolvidos (ALMEIDA e MISHIMA, 2001).

O objetivo do presente estudo é, refletir sobre o entendimento de que o trabalho em equipe, constitui a base dessa proposta de mudança na reforma do Sistema de Saúde brasileiro. Este entendimento, faz-se necessário, uma vez que a articulação entre os profissionais envolvidos e a comunidade, possam criar uma nova cultura e, o que garanta uma prática pautada nos princípios da promoção da saúde.

Ainda há poucos estudos sobre esta estratégia inovadora que é o Programa Saúde da Família. Utilizamos como algumas das referências para este trabalho, os estudos de Peduzzi (1998) e Araújo (2004).

\section{Modelo Assistencial e possibilidades futuras}

As discussões sobre mudança de práticas na saúde, na época da reestruturação dos serviços de saúde, por volta dos anos 90, estavam muito mais voltadas para o nível do aparato legal e da estrutura políticoadministrativa do SUS, como elementos de maior destaque na conjuntura política. Essa é apenas uma constatação do elenco de prioridades político-institucionais que alimentavam o movimento reformador e o debate da ocasião, sem negar todo o acúmulo e a importância das discussões sobre aparelho formador e o SUS, perfil do novo profissional para o sistema, integração docente-assistencial, etc., entre outras contribuições.

É inegável, porém, que não havia uma preocupação maior com relação aos atores que concretamente operariam essas mudanças. Nesse sentido, um dos fatos críticos de maior destaque no Brasil dos últimos 20 anos era justamente a sempre adiada conferência nacional específica dos recursos humanos da área.

Profissionais e pacientes ainda encontravam-se em patamares separados, enquanto equipe-comunidade. Esta situação se reproduzia tanto no setor público quanto no setor privado. Segundo Campos (1992), estruturar serviços com base nas equipes de saúde contribui para a quebra da divisão do processo de trabalho, para a responsabilização pelos problemas apresentados e para a superação da inércia e indiferença burocrática dos serviços públicos de saúde. 
Nas unidades de Saúde, predominava a hegemonia do poder técnico e político dos profissionais médicos, em conflito com outros profissionais de nível universitário. As categorias de nível médio eram mais numerosas, embora menos qualificadas e mais desvalorizadas em termos salariais (MOURA, 1994).

Em geral, o trabalho é feito de forma fragmentada e ainda não se correlaciona a finalidade de seu trabalho específico com o objetivo do trabalho em saúde. Moura (1994), salienta que discutir mudanças nas práticas de saúde visando à redefinição do modelo de atenção, implica em abordar a forma ou o modo como essas práticas são produzidas e traduzidas em ações de saúde.

De uma maneira geral, tanto a sociedade mostrava-se indiferente à situação crítica do setor saúde, como também, os profissionais que muitas vezes adotavam posturas alienadas (CAMPOS, 1994).

Vejamos agora um conjunto de principais definições governamentais (através do Ministério da Saúde) sobre as mudanças preconizadas pelo PSF, iniciadas em meados dos anos 90, e que são fundamentais para a compreensão deste estudo, nesse novo modelo de atenção que vem sendo implementado na atualidade da saúde brasileira: A partir de 1996, com a edição da NOB/96 (BRASIL, 1996), cristaliza-se a necessidade de redefinição do modelo de saúde, adotando como estratégia principal a ampliação de cobertura do PSF/PACS. Assim, para uma nova estratégia, faz-se necessário um novo profissional.

Se a formação dos profissionais não for transformada no aparelho formador, o modelo de atenção também não o será na realidade do dia-a-dia (LEVCOVITZ e GARRIDO, 1996).

Segundo orientações do Ministério da Saúde (BRASIL, 2001), no PSF as ações são estruturadas no trabalho em equipe e buscam humanizar as práticas de saúde, com o objetivo de obter a satisfação do usuário através do estreito relacionamento dos profissionais com a comunidade. O processo de trabalho das equipes se estrutura a partir do conceito de delimitação do território, mapeamento das áreas e micro-áreas (a primeira entendida como um conjunto de micro-áreas contíguas sob a responsabilidade de uma equipe, onde residem em torno de 2.500 a 4.500 pessoas e a segunda representa a área de atuação de um agente comunitário de saúde, onde moram cerca de 400 a 750 pessoas, com cadastradascadastramento familiar e utilização do sistema de informação de atenção básica (SIAB).

As principais responsabilidades atualizadas, de acordo com a NOAS/2001 (BRASIL, 2001), constituem: saúde da criança; saúde da mulher; controle de hipertensão; controle de diabetes melittus; controle de tuberculose; eliminação da hanseníase; ações de saúde bucal. Somam-se a isso visitas domiciliares, reuniões entre os profissionais e a comunidade, atividades educativas com grupos definidos, ações administrativas de supervisão e educação continuada. Seguindo essas diretrizes, uma equipe de saúde da família deve ser composta minimamente de um médico, um enfermeiro, um auxiliar de enfermagem e quatro ou seis agentes comunitários de saúde. A incorporação de outras categorias profissionais dependerá dos municípios. Cada equipe deve ser responsável pela cobertura de 600 a 1.000 famílias.

Dentre as atividades da equipe de saúde estariam: conhecer a realidade das famílias; identificar os problemas de saúde e situação de risco; realizar o planejamento e programação local com a participação comunitária; estabelecer vínculo de confiança com os usuários através de uma conduta ética; resolver os problemas de saúde em nível de atenção básica; garantir o acesso à comunidade dentro de um tratamento de referência e contra-referência; prestar atendimento integral à demanda, respondendo à esta de forma contínua e racionalizada; coordenar e/ou participar de grupos de educação em saúde; promover ações intersetoriais e outras parcerias com organizações formais e informais existentes na comunidade para o enfrentamento conjunto dos 
problemas identificados; fomentar a participação popular, discutindo com a comunidade conceitos de cidadania, de direito à saúde e suas bases legais; incentivar a participação ativa da comunidade nos conselhos locais de saúde, no conselho municipal de saúde e auxiliar na implantação do cartão nacional de saúde.

A criação da Estratégia Saúde da Família, com a implantação do PSF por si só não garante a modificação do modelo assistencial médico-centrado. Esta depende de uma mudança na forma de se produzir o cuidado, assim como dos diversos modos de agir dos profissionais entre si e com os usuários. $\mathrm{O}$ "campo do cuidado" e a interação abrem a possibilidade de cada um usar todo o seu potencial criativo e criador na relação com o usuário, para juntos realizarem a produção do cuidado (CAMPOS, 1994).

Segundo Merhy (1997)

As especificidades do trabalho em saúde demarcam um campo específico das práticas, sendo que estas se desenvolvem em um espaço de encontro e de intervenção denominado de espaço intercessor, enquanto espaço onde se processa a produção de ações de saúde e onde cada trabalhador potencialmente pode decidir coisas, ou seja, exerce um certo autogoverno.

A operação cotidiana desse autogoverno, do estabelecimento de espaços intercessores na produção de atos de saúde se dá através do "trabalho vivo em ato" aquele que ocorre no momento mesmo em que ele se realiza, no imediato fazer da produção do serviço. A mudança assistencial dar-se-ia a partir da reorganização do processo de trabalho estruturado na ação multiprofissional.

Merhy (1997), menciona ainda que esses novos fazeres e práticas se materializam em tecnologias de trabalho usadas para produzir a saúde, que são traduzidas a partir dos conhecimentos e agires aplicados à produção de algo e que se classificam em duras e leve-duras, ou lugares do "trabalho morto". Por outro lado, os conhecimentos podem estar diluídos nas experiências e modos singulares de cada profissional operar o seu "trabalho vivo" que está inscrito no campo das tecnologias leves, fundamentais ao trabalho em saúde ${ }^{18}$. O aprisionamento de cada um em seu campo específico de saberes e práticas limita o processo de trabalho às estruturas rígidas do conhecimento técno-estruturado, tornando-o trabalho "morto-dependente". Para que o trabalho em equipe seja viabilizado, há necessidade de uma relação interativa entre os trabalhadores, mediada pela troca de conhecimentos e articulação de um "campo de produção do cuidado" comum a todos (FRANCO e MERHY, 1998).

Segundo Peduzzi (1998), o trabalho em equipe é uma modalidade do trabalho coletivo, sendo caracterizado pela relação recíproca entre as dimensões complementares de trabalho e interação. Nesse estudo, observa-se que, apesar das escassas definições sobre equipe de saúde, na literatura foram encontradas diferentes abordagens sobre esse tema, onde se destacam: os resultados, as relações e a interdisciplinaridade. Na lógica dos resultados, a equipe é concebida como um recurso para o aumento da produtividade e da racionalização dos serviços. Nas relações, as pesquisas utilizam como referência os conceitos da psicologia analisando as equipes com base nas relações interpessoais, assim como nos processos psíquicos. Na perspectiva da interdisciplinaridade, situam-se os trabalhos que trazem à discussão a articulação de saberes e a divisão do trabalho, ou seja, da especialização do trabalho em saúde.

Quanto as diversidades sobre à concepção dos agentes (profissionais de saúde) acerca do trabalho em equipe, observa-se que, para os médicos, a equipe é vista como sendo equipe médica. Outros trabalhadores de nível superior defendem a construção de uma equipe multiprofissional e referem ainda a dificuldade em 
constituí-la por sentirem falta de espaço dos "não-médicos" para exercício de suas funções. Quanto aos de nível* médio, demonstraram pouca preocupação com essa situação, uma vez que se consideram bem definidos nas suas funções, apontam apenas a dificuldade das relações de subordinação com as chefias imediatas (PEDUZZI, 1998).

Diante da diversidade das concepções de trabalho em equipe, a idéia de equipe perpassa duas concepções distintas: a equipe como agrupamento de agentes e a equipe como integração entre relações e práticas. A primeira é caracterizada pela fragmentação das ações e a segunda, pela construção de possibilidades de recomposição. Esta última estaria consoante com a proposta da integralidade das ações de saúde e a necessidade contemporânea de recomposição dos saberes e trabalhos especializados.

Com relação ao trabalho de equipe, um elemento de forte consenso entre os autores está no fato de que o trabalho em equipe implica o compartilhar do planejamento, a divisão de tarefas, a cooperação e a colaboração. A interação democrática entre diferentes atores, saberes, práticas, interesses e necessidades representam a possibilidade do novo no trabalho em equipe (FEUERWEKER e SENA, 1999).

Segundo Piancastelli, Faria e Silveira (2000), a dificuldade do trabalho em equipe está relacionada com as diferentes concepções sobre o conceito de equipe. Dentre essas, se observam as definições: a equipe como um conjunto ou grupo de pessoas que desempenham uma tarefa ou trabalho não importando, nesse caso, os objetivos e as relações interpessoais; ou a equipe como um grupo de pessoas que tem um objetivo comum. Sendo, nesse caso, fundamental que tenham o mesmo objetivo, não importando como cada um pretenda alcançá-lo; ou a equipe como um conjunto de pessoas que além de um objetivo comum pretendem alcançá-lo de forma compartilhada; ou refere-se ainda à equipe como um conjunto ou grupo de pessoas cujo objetivo é resultante da negociação/discussão entre todos os membros do grupo e por fim com o conceito que considera equipe como um grupo de pessoas com habilidades complementares, comprometidas umas com as outras e pelo objetivo comum, obtido através da negociação entre os atores sociais envolvidos no plano de trabalho.

Cianpone e Peduzzi (2000), afirmam que, no processo de produção em saúde, a denominação "equipe" sempre fará referência a uma situação de trabalho, e este, referindo-se à obtenção de bens ou produtos para a atenção às necessidades humanas. Além disso, destacam as habilidades relacionais tomadas como ferramentas essenciais da proposta e transformação de modelos assistenciais. Dessa forma, na Estratégia Saúde da Família, o trabalho em equipe constitui uma prática em que a comunicação entre os profissionais deve fazer parte do exercício cotidiano do trabalho, no qual os agentes operam a articulação das intervenções técnicas por meio da linguagem.

Essa articulação, na equipe multiprofissional, refere-se à recomposição de processos de trabalhos distintos e, portanto, deve considerar as conexões e interfaces existentes entre as intervenções peculiares de cada área profissional, preservando as respectivas especificidades. Peduzzi (1998) chama atenção ainda para o fato de a formação dos profissionais de saúde estar pautada no modelo biomédico, o que pode representar uma limitação para a atuação na esfera da interação, entendida como uma prática comunicativa por meio da qual os envolvidos buscam o consenso quanto a um projeto comum (HABERMAS, 1989).

Mishima et al. (2000) orienta que o estabelecimento de uma relação dialógica no interior das unidades de saúde pode contribuir para a superação de relações hierarquizadas, em que os profissionais raramente conhecem as potencialidades dos outros, reproduzindo, dessa forma, a divisão social do trabalho e estabelecendo relações de mando e autoridade. 
Nesse sentido, concordamos com Paim e Almeida Filho (2000) ao afirmarem que um profissional de saúde, e em especial de saúde pública, necessita das seguintes características,

Capacidade de análise do contexto em relação às práticas que realiza; compreensão da organização e gestão do processo de trabalho em saúde; exercício de um agir comunicativo ao lado do pensamento estratégico; advocacy ou habilidade para proceder denúncia de situações e convencimento de interlocutores; tolerância e diálogo em situações de conflitos; atenção a problemas e necessidades de saúde; senso crítico quanto à efetividade e ética das intervenções propostas ou realizadas e finalmente permanente questionamento sobre o significado e o sentido do trabalho e dos projetos de vida.

Assim, são importantes as considerações de Motta (2001) ao demonstrar que tecnologias importantes na qualificação da assistência como a interação, o trabalho em equipe, o planejamento situacional e a comunicação são pouco valorizadas pelos diferentes níveis gerenciais. Os novos modelos de organização da assistência buscam estimular a construção de equipes, ao permitirem a recomposição dos meios de trabalho, a reestruturação das atividades dos agentes e a redefinição das relações sociais e técnicas sob as quais se realizam os trabalhos.

A concepção de trabalho em equipe está vinculada à de processo de trabalho e vem, portanto, sofrendo transformações ao longo do tempo. Historicamente, surge da necessidade do homem de somar esforços para alcançar objetivos de forma mais fácil e de atender às exigências do processo de produção moderno.

O trabalho em equipe é uma forma eficiente de estruturação, organização e de aproveitamento das habilidades humanas. Possibilita uma visão mais global e coletiva do trabalho, reforça o compartilhamento de tarefas e a necessidade de cooperação para alcançar objetivos comuns (MOTTA, 2001). Se não houver interação entre os profissionais das equipes de Saúde da Família, corre-se o risco de repetir a prática fragmentada, desumana e centrada no enfoque biológico individual com diferente valoração social dos diversos trabalhos

(ALMEIDA E MISHIMA, 2001).

\section{O Sistema de colaboração mútua entre os atores sociais}

Diante da situação de crise no setor saúde no Brasil, ao longo dos anos têm sido propostos novos modelos assistenciais com uma forte tendência de organizar as práticas de saúde no trabalho interdisciplinar, com o objetivo de atender em quantidade e qualidade e de forma equânime as demandas da população.

A interdisciplinaridade não representa a anulação da disciplinaridade, nem a especificidade de cada saber, mas a possibilidade de uma compreensão integral do ser humano e do processo saúde-doença, objeto do trabalho em saúde, passa necessariamente por uma abordagem interdisciplinar e por uma prática multiprofissiona (FEUERWEKER e SENA, 1999).

A construção da interdisciplinaridade tem sido um processo contínuo e crescente no setor saúde, dada a necessidade de superação da fragmentação do conhecimento humano, na busca de uma visão globalizada que dê conta da complexa dimensão do processo saúde-doença.

O conceito de saúde como qualidade de vida requer a passagem de um trabalho individual, compartimentado para uma ação coletiva. Supõe a compreensão de pensamentos e ações desiguais e pressupõe a existência de 
pontos comuns entre pessoas que pretendem desempenhar uma ação interdisciplinar (WESTPHAL e MENDES, 2000).

Leite (2001) considera que a interdisciplinaridade representa uma possibilidade de aproximação ou estreitamento entre as partes, uma vez que a totalidade nunca pode ser atingida. Faz-se necessária, portanto, a adoção de uma visão dialética dos obstáculos de sua construção. $\mathrm{O}$ autor afirma que disciplina tem o mesmo sentido que ciência, instrução, ensino. O prefixo "inter" está relacionado com troca, reciprocidade. Assim, interdisciplinar refere-se à troca entre áreas de conhecimento. Para atingir esses objetivos, deve-se estabelecer entre os membros da equipe uma nova concepção de trabalho que admita a diversidade de ação, busque constantemente o consenso e que esteja baseada na interdisciplinaridade.

O trabalho interdisciplinar tem como significado: a possibilidade de a prática de um profissional se reconstruir na prática do outro, transformando ambas na intervenção do contexto onde estão inseridas (BRASIL, 2001).

\section{Considerações finais}

O trabalho em equipe no contexto do PSF ganha uma nova dimensão no sentido da divisão de responsabilidades do cuidado entre os membros da equipe, na qual todos participam com suas especificidades contribuindo para a qualidade da prestação das ações de saúde. A concepção integral do cuidado favorece a ação interdisciplinar nas práticas, onde a valorização das diversas disciplinas contribui para uma abordagem ampla e resolutiva do cuidado. Nessa perspectiva, a responsabilidade da atenção passa a ser descentralizada da figura do profissional médico, considerado o centro da equipe no modelo hospitalocêntrico, sendo dividida entre os membros da equipe.

A importância do diálogo na busca do consenso constitui elemento imprescindível para o bom desenvolvimento do trabalho em equipe. Isso aponta à possibilidade do desenvolvimento de uma prática comunicativa. O trabalho em equipe "provoca" a escuta do outro. O que pressupõe o estabelecimento de um canal de comunicação.

O processo de trabalho em saúde está fundamentado numa inter-relação pessoal forte onde os conflitos também estão presentes no dia-a-dia da equipe (NOGUEIRA, 2000). Deve-se considerar ainda que uma equipe é composta por pessoas que trazem especificidades próprias como: gênero, inserção social, tempo e vínculo de trabalho, experiências profissionais e de vida, formação e capacitação, visão de mundo, diferenças salariais e, por fim, interesses próprios. Essas diferenças exercem influência sobre esse processo de trabalho, uma vez que estão presentes no agir de cada profissional, mas não inviabilizam o exercício da equipe. A mudança nas relações de trabalho não acontecerá de maneira rápida, os profissionais que compõem as equipes vêm de uma prática na qual predomina o poder do nível superior sobre o nível médio, da categoria médica sobre as demais. Nesse contexto, relações de poder hierarquizadas, estabelecidas entre os profissionais, configuram elementos que fortalecem a situação de status de algumas profissões sobre outras, garantindo posições de liderança na equipe.

Dessa forma, se faz necessário redefinir no cotidiano das USFs responsabilidades e competências dos integrantes da equipe de saúde, sem esquecer os pontos de interseção entre as disciplinas. 
O Clima Organizacional, isto é, a forma como as pessoas vivem seus problemas no interior dos serviços implica o estabelecimento de interações, onde faz-se necessária uma aproximação maior entre os sujeitos que prestam o cuidado. Ou seja, estabelecer uma prática comunicativa como estratégia para o enfrentamento dos conflitos significa romper com velhas estruturas hierarquizadas, tão presentes no modelo de saúde hegemônico. Nessa realidade, os profissionais de saúde desenvolvem o trabalho com relativa autonomia, mas com subordinação ao fazer do médico, ao contrário do que acontece na ESF, em que as relações de poder se enfraquecem quando se prioriza a escuta. A ação comunicativa é contrária a qualquer tipo de repressão dos direitos à liberdade do sujeito. Torna-se essencial que os profissionais se relacionem em um ambiente livre de coações, para que juntos se comuniquem, estabelecendo interação, possibilitando assim a construção de um novo modelo de saúde.

\section{Referências}

ALMEIDA MCP, MISHIMA SM. O desafio do trabalho em equipe na atenção à Saúde da Família: construindo "novas autonomias" no trabalho. Interface comunicação, saúde, educação 2001; 9:150-153.

ALMEIDA FILHO N. Intersetorialidade, transdisciplinaridade e saúde coletiva: atualizando um debate em aberto. Revista de Administração Pública 2000; 34(b):11-34.

ARAÚJO MB. A Equipe de Saúde no PSF: mudando práticas? Estudo de Caso no município de Natal/RN [dissertação]. Natal (RN): Departamento de Enfermagem da Universidade Federal do Rio Grande do Norte; 2004.

ARTMANN E. Interdisciplinaridade no enfoque intersubjetivo habermasiano; reflexões sobre planejamento e AIDS. Rev C S Col 2001; 1:183-195.

BRASIL. Ministério da Saúde. Departamento de Atenção Básica. Guia prático do programa de saúde da família. Brasília; 2001.

BRASIL. Ministério da Saúde. Norma Operacional Básica SUS 01/1996. Gestão Plena com Responsabilidade pela Saúde da População. Portaria MS/GM N. ${ }^{\circ}$ 2.203/1996.

CAMPOS FE, BELISÁRIO SA. O Programa de Saúde da Família e os desafios para a formação profissional e a educação continuada. Interface comunicação, saúde, educação 2001; 9:133-141.

CAMPOS GWS. Reforma da Reforma: repensando a saúde. São Paulo: Hucitec; 1992.

CAMPOS GWS. Considerações sobre a arte da ciência da mudança: revolução de coisas e reforma das pessoas. O caso da saúde. In: Cecílio LCO, organizador. Inventando a mudança na saúde. São Paulo: Hucitec; 1994. p.29-87.

CECÍLIO LCO. As necessidades de saúde como conceito estruturante na luta pela integralidade e eqüidade na atenção em saúde. In: Pinheiro R, Mattos RA, organizadores. Os sentidos da integralidade na atenção e no cuidado à saúde. Rio de Janeiro: IMS-UERJ/ABRASCO; 2001. p. 113-126.

CIAMPONE MHT, PEDUZZI M. Trabalho em equipe e trabalho em grupo no Programa de Saúde da Família. Revista Brasileira de Enfermagem 2000; 53: 43-147.

FEUERWEKER LCM, SENA RR. Interdisciplinaridade, trabalho multiprofissional e em equipe. Sinônimos? Como se relacionam e o que têm a ver com nossa vida? Revista Olho Mágico 1999; 5:18.

FRANCO TB, MERHY EE. PSF: Contradições de um programa destinado a mudanças de um modelo tecno-assistencial. Campinas: UNICAMP; 1998. [Mimeo].

FRANCO TB, MERHY EE. PSF: contradições e novos desafios. In: Conferência Nacional de Saúde. Tribuna Livre 2000. [acessado 2000 Abr 28]. Disponível em: http://www.datasus.gov.br/cns/temas/tribuna.htm

GOMES R, DESLANDES SF. Interdisciplinaridade na saúde pública: um campo em construção. Revista Latino-Americana de Enfermagem 1994; 2 (2):103-114.

HABERMAS J. Consciência moral e agir comunicativo. Rio de Janeiro: Tempo Brasileiro; 1989.

KESSELRING T. De onde provêm as normas éticas? Da decisão individual ou do discurso livre? A controvérsia entre Habermas e Tugendhat. In: De Boni LA, organizador. Finitude e transcendência. Petrópolis: Vozes; 1996. p. 766-781.

LEITE JCA. $O$ trabalho da enfermeira na equipe de saúde da família: em busca da interdisciplinaridade [dissertação]. Belo Horizonte (MG): Escola de Enfermagem/UFMG; 2001.

LEVCOVITZ E, GARRIDO NG. Saúde da família: a procura de um modelo anunciado. Cadernos de Saúde da Família 1996; 1(1): 3-7. 
MATTOS RA. Os sentidos da integralidade: algumas reflexões acerca dos valores que merecem ser defendidos. In: PINHEIRO R, MATTOS RA, organizadores. Os sentidos da integralidade na atenção e no cuidado à saúde. Rio de Janeiro: IMS- UERJ/ABRASCO; 2001. p. 39-64.

Integralidade e a formulação de políticas específicas de saúde. In: Pinheiro R, Mattos RA, organizadores. Construção da integralidade: cotidiano, saberes e práticas de saúde. Rio de Janeiro: IMSUERJ/ABRASCO; 2003. p. 45-59.

MERHY EE. Em busca do tempo perdido: a micropolitica do trabalho vivo em saúde. In: Merhy EE, Onocko R, organizadores. Agir em saúde: um desafio para o público. São Paulo: Hucitec; 1997. p.71-112.

MISHIMA SM, et al. O velho trabalho em equipe pode ganhar cara nova? Uma perspectiva do trabalho gerencial. Saúde em Debate 2000; 54:66-74.

MOTTA RP. Desempenho em equipes de saúde: Manual. Rio de Janeiro: Editora FGV; 2001.

MOURA RMF. O processo de trabalho da equipe multiprofissional em centros de saúde: (vi) vendo e indagando. Anais do Congresso da ABRASCO, 1994; Olinda/PE.

NUNES ED. Interdisciplinaridade: conjugar saberes. Saúde em Debate 2002; 26(62):249-258.

NOGUEIRA RP. O trabalho nos serviços de saúde. In: Santana JP, organizador. Organização do cuidado a partir de problemas: uma alternativa metodológica para a atuação da equipe de saúde da família. Brasília: OPAS/Representação do Brasil; 2000. p. 59-63.

PAIM JS. Saúde da família: espaço de reflexão e de contra-hegemonia. Interface comunicação, saúde, educação 2001; 9:143-145.

PAIM JS, ALMEIDA FILHO N. A crise na saúde pública e utopia da saúde coletiva. Salvador: Casa da Qualidade Editora; 2000.

PEDUZZI M. Equipe multiprofissional de saúde: a interface entre o trabalho e interação [tese]. Campinas (SP): Faculdade de Ciências Médicas, Universidade Estadual de Campinas; 1998.

PIANCASTELLI CH, FARIA HP, SILVEIRA MR. O trabalho em equipe. In: Santana JP, organizador. Organização do cuidado a partir de problemas: uma alternativa metodológica para a atuação da equipe de saúde da família. Brasília: OPAS/Representação do Brasil; 2000. p 45-50.

PINHEIRO R, LUZ MT. Práticas eficazes x modelos ideais: ação e pensamento na construção da integralidade. In: PINHEIRO R, MATTOS RA, organizadores. Construção da integralidade: cotidiano, saberes e práticas de saúde. Rio de Janeiro: IMS-UERJ/ABRASCO; 2003. p. 7-34.

ROCHA PM. No limiar do século XXI: globalização e saúde, desafios da proteção social no Brasil dos anos 90 [tese]. Rio de Janeiro (RJ): IMS-UERJ; 1997.

WESTPHAL MF, MENDES R. Cidade saudável: uma experiência de interdisciplinaridade e intersetorialidade. Revista de Administração Pública 2000; 34(b):47-61.

Sobre os autores:

(1) Lívia Arcanjo Fonteles é graduada em Medicina pela Universidade Federal do Ceará.

E-mail: liviaarcanjo84@ hotmail.com

(2) Francisca Ergovânia Batista de Brito é Bacharel em Administração de Empresa pela Faculdade Dr. Leão Sampaio em Juazeiro do Norte - CE. Concludente do curso de Enfermagem pela mesma Instituição. Especialista em Adm. Hospitalar pela (URCA). Especializanda em Políticas Públicas em Saúde Coletiva (URCA). Técnica em Segurança e Saúde no Trabalho-SST (IBTF-Instituto Brasileiro de Educação e Tecnologia de Formação). Especializanda em Enfermagem do Trabalho (UVA).

E-mail: ergovaniabrito@hotmail.com

(3) Emmanuel Nunes Sampaio de Lacerda é Acadêmico de Medicina pela Universidade Federal do Ceará - UFC.

E-mail: emmanuelnsl@bol.com.br

(4) Agrênia Custódio de Lima é Acadêmica de Enfermagem pela Faculdade de Juazeiro do Norte - FJN. E-mail: agrenia.a@hotmail.com

Como citar este artigo (Formato ISO):

FONTELES, L.A., BRITO, F.E.B e LACERDA, E.N.S, LIMA, A.C. A estratégia do Programa Saúde da Família. Id on Line Revista de Psicologia, Fevereiro de 2011, vol.1, n.13, p.11-19. ISSN 1981-1189. 